\title{
Long-term sustainability of zero central-line associated bloodstream infections is possible with high compliance with care bundle elements
}

\author{
E. Hakko, ${ }^{1}$ S. Guvenc, ${ }^{2}$ I. Karaman, ${ }^{3}$ A. Cakmak, ${ }^{2}$ T. Erdem ${ }^{2}$ and M. Cakmakci ${ }^{4}$
}

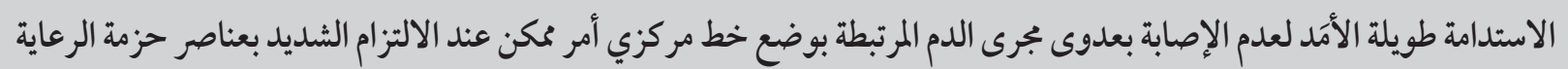

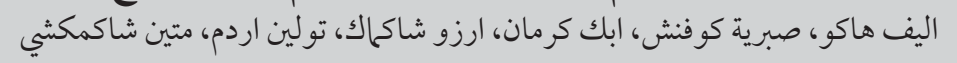

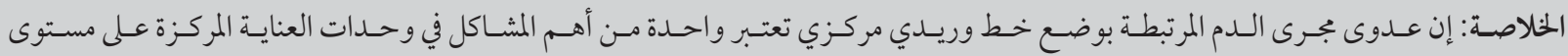

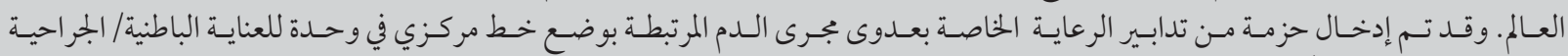

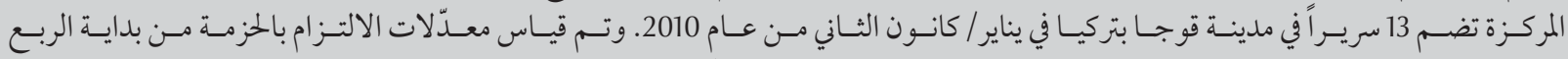

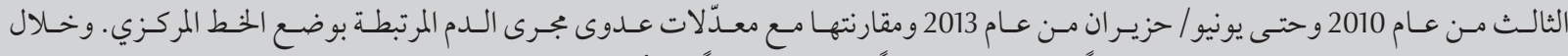

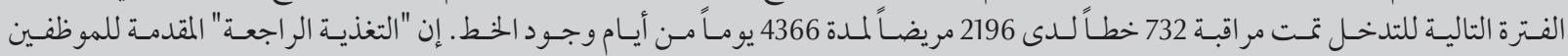

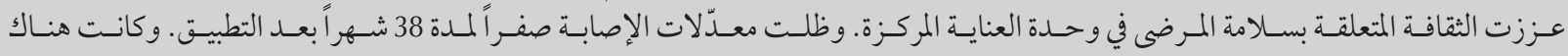

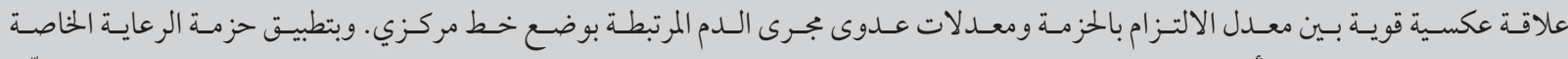

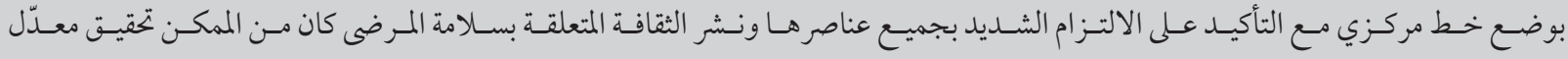

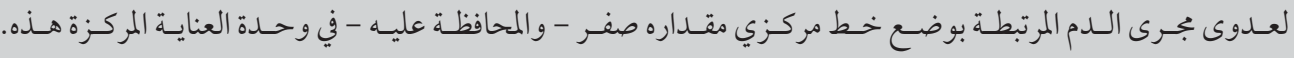

ABSTRACT Central-line-associated bloodstream infection (CLABSI) is one of the most important problems in intensive care units (ICUs) worldwide. A bundle of CLABSI care measures was introduced at a 13-bed medical/ surgical ICU in Kocaeli, Turkey in January 2010. Compliance rates with the bundle were measured at the beginning of the third quarter of 2010 until June 2013 and compared with CLABSI rates. During the post-intervention period, of 2196 ICU patients, 732 lines placed for 4366 line-days were monitored. Feedback to staff reinforced a culture of patient safety in the ICU. Infection rates remained zero for 38 months after the implementation. There was a strong negative correlation between bundle compliance rate and CLABSI rates. With the implementation of the central-line bundle of care, together with emphasis on high compliance with all its components and a culture of patient safety, it was possible to achieve and maintain a zero rate of CLABSI in this ICU.

La pérennité d'un taux zéro d'infection sur cathéter central est rendue possible par un haut niveau d'application des éléments de l'ensemble de soins

RÉSUMÉ Les infections sur cathéter central sont l'un des problèmes les plus importants dans les unités de soins intensifs au niveau mondial. Un ensemble de soins dispensés en cas d'infections sur cathéter central a été introduit dans une unité de soins intensifs médicaux et chirurgicaux de 13 lits à Kocaeli (Turquie) en janvier 2010. Le degré d'application de l'ensemble de soins a été évalué du début du troisième trimestre 2010 jusqu'à juin 2013 et comparé aux taux d'infections sur cathéter central. Au cours de la période suivant l'intervention, parmi les 2196 patients ayant séjourné dans l'Unité des soins intensifs, 732 cathéters centraux posés pendant 4366 jours cumulés ont été soumis à observation. Le retour d'information fourni au personnel a permis de renforcer la culture de la sécurité du patient en unité de soins intensifs. Les taux d'infections sont restés nuls pendant les 38 mois ayant suivi la mise en œuvre. Il existait une forte corrélation négative entre le degré d'application del'ensemble de soins et les taux d'infections surcathéter central. La mise en œuvre de cet ensemble de soins concernant les cathéters centraux, associée à l'accent mis sur le degré d'application de toutes ses composantes et une culture de la sécurité du patient, a permis d'atteindre et de maintenir un taux zéro d'infection sur cathéter central dans cette unité de soins intensifs.

${ }^{7}$ Department of Infectious Diseases; ${ }^{2}$ Intensive Care Unit; ${ }^{3}$ Infection Control Department; ${ }^{4}$ Department of General Surgery, Anadolu Medical Centre, Gebze, Kocaeli, Turkey (Correspondence to E. Hakko: elifhakko@gmail.com).

Received: 19/08/14; accepted: 03/02/15 


\section{Introduction}

Central-line-associated bloodstream infection (CLABSI) is one of the most important problems in intensive care units (ICUs) worldwide. Such infections are associated with a high morbidity rate and excess utilization of medical resources. In the United States alone, there are an estimated 80000 catheter-related bloodstream infections, with 28000 deaths every year. The average cost of care for a single patient with this infection is approximately US\$ 45000 and the total annual expenditure is around US\$ 2.3 billion (1). CLABSI is associated with increased length of ICU and hospital stays by 2.4 and 7.5 days, respectively (2). According to the National Healthcare Safety Network (NHSN) 2004 report, the median rate of CLABSI in ICUs of all types ranges from 1.8 to 5.2 per 1000 catheter-days. However, the NHSN benchmarks continue to decrease the rates to an ultimate goal of near zero (3). The call for elimination of CLABSI has gained international attention $(4,5)$.

Reported intervention studies have shown success in reduction of CLABSI rates but few have reported long-term improvements. Recently, interventions for control of CLABSI have been formulated into a so-called "bundle" of recommendations for care (6) that helps providers to deliver more consistent care by giving a set structure to an event with all the steps being "all-or-nothing" events which are either performed or not performed (7). With the goal in mind, we implemented a bundle of care in the medical/surgical ICU in a hospital in Turkey. This paper reports how we achieved and sustained a zero rate of CLABSI for 3 years after the immediate post-intervention period.

\section{Methods}

\section{Study design and setting}

This was a 4-year observational cohort study at Anadolu medical centre, which is a 209-bed, non-profit private hospital in Gebze district of Kocaeli province, Turkey. It includes a 13-bed medical/ surgical ICU. The nursing to patient ratio is on average $1: 2$ and rarely $1: 3$.

\section{Patients}

All patients who were admitted to the ICU from January 2010 to December 2013 and who received a central venous catheter were prospectively included in the study. The Centers for Disease Control and Prevention case definition for CLABSI was used: 1) patient has a recognized pathogen cultured from one or more blood cultures, and the organism cultured from blood is not related to an infection at another site; 2) patient has at least one of the symptoms fever $\left(>38^{\circ} \mathrm{C}\right)$, chills or hypotension; positive laboratory results not related to an infection at another site; and a common skin contaminant (i.e. diphteroids [Corynebacterium spp.]. Bacillus spp. [not B. anthracis], Propionibacterium spp., coagulase-negative staphylococci [including S. epidermidis], viridans group streptococci, Aerococcus spp. or Micrococcus spp.) is cultured from 2 or more blood cultures drawn on separate occasions.

For this study blood cultures were drawn if the patient had fever $\left(38^{\circ} \mathrm{C}\right)$, chills or hypotension. At least 2 vials were taken: one from the central line, the other from a peripheral vessel. If blood yielded a microorganism and the patient had no other infection at another site the case was considered as a central-line infection. Additionally, if blood taken from the central line was positive earlier than the other vial, we strongly accepted the case as CLABSI. According to our hospital infection control programme, 2 sets of blood cultures are taken from every patient who has fever before starting empirical antibiotics.

In this study period, 4290 blood cultures were obtained per 4366 centralline days (98\%) from 2196 patients (i.e. a blood culture set of approximately 2 vials per patient).

\section{Intervention}

A multidisciplinary team was formed to implement the central-line care bundle. The team included the head physician of the ICU, the infectious diseases physician, the nurse manager of the unit and an infection control nurse. The target was to reduce the CLABSI rate towards near-zero in the ICU. Infection control strategies that were previously used for the prevention of CLABSI were put in the bundle protocol. All health-care workers including physicians, nurses and technicians were trained about this protocol. The bundle was introduced in January 2010 and from the third quarter of 2010, the nurse manager of the ICU and the infection control nurse checked the compliance with the bundle by using a central-line chart daily. We measured the compliance rates for every element but we accepted full compliance if every element suitable for this patient performed simultaneously assuming the "all or nothing" principle.

The bundle included the following 4 elements: 1) removal of all lines placed in the emergency room or in another hospital within 24 hours, assuming that compliance to the maximum barrier precautions were not $100 \%$ in that setting; 2) use of aseptic technique, hand hygiene and maximum barrier precautions (caps and masks, surgical scrub, sterile gown and gloves); 3 ) use of a dedicated lumen for total parenteral nutrition; and 4) total parenteral nutrition infusion sets changed in 24 hours.

Other infection control precautions regarding CLABSI were: 1 ) hand hygiene before and after each procedure; 2) avoiding femoral site catheter insertion; 3) review of central lines on a daily basis; 4) evaluation of the insertion site for symptoms of infection twice daily; and 5) covering the catheter insertion site with a transparent dressing and not changing it unless it became soiled.

\section{Data analysis}

The rate of infection was calculated as follows: (number of CLABSIs/number 
of central-line days) $\times 1000$ for each month and quarterly. Device utilization ratio was calculated as follows: number of device or catheter-days/number of patient-days.

SPSS version 17.0 was used to perform the data analysis. The relationship between parameters was analysed with scatter-plot and the results showed that distribution was both monotonic and linear. Not all variables were normally distributed as assessed by the ShapiroWilk test. Non-parametric Spearman correlation test was performed to determine any association between bundle compliance rate and CLABSI rate.

\section{Results}

\section{Pre-intervention}

Our hospital began providing patient care in 2005. The rate of CLABSI that year was 12.8 per 1000 catheter-days. From 2006 to 2010, the CLABSI rates ranged from 1.6 to 22.9 per 1000 catheter-days. The run chart of quarterly rates of CLABSI in relation to the infection control strategies are shown in Figure 1.

From the blood cultures Pseudomonas spp., Burkholderia cepacia, Enterococcus faecalis, methicillin-resistant Staphylococcus aureus, Escherichia coli, Streptococcus pyogenes, coagulase negative staphylococcus, Acinetobacter spp., Stenotrophomonas maltophilia were found. We did not have any outbreaks.
In 2005, our efforts were on education of the medical staff. However, we had a peak CLABSI rate in 2006, corresponding with a high usage of femoral catheters. With avoidance of femoral catheters the rate decreased from 22.9 to 5.93 per 1000 catheter-days. We were still not on target and so a strategy was implemented of checking the necessity of the catheters and daily compliance with maximum barrier precautions. Between 2007 and 2010 efforts were made to raise the awareness of the staff about these issues and to re-educate newly appointed nurses. Hand hygiene campaigns were promoted. Nevertheless, we could not eliminate CLABSIs from the ICU and decided to implement a bundle of infection control measures.

\section{Post-intervention}

In January 2010, we introduced the bundle of care for CLABSI prevention. During the post-intervention period reported here (2010-13), a total of 732 lines were placed in 2196 patients for 4366 line-days. Of these lines, $73.6 \%$ were jugular, $24.9 \%$ subclavian, $1.5 \%$ femoral and $100 \%$ multiple lumen. The lines stayed in for a range of 2-28 days, mean 5.9 days. The overall mean length of ICU stay was 4.6 days but the length of stay was greater in patients with central lines because patients who had a central line were mostly nonsurgical patients. The mean of length of ICU stay in these patients was 13.4 days. Patients were Turkish residents and patients coming from different countries such as Romania, Bulgaria, Iraq, Libya, Russia, Kazakhstan and Turkmenistan.

We commenced periodic measurements of the rate of compliance with the bundle in the month of May 2010 because we wanted to see if this new intervention was accepted by health-care workers. The compliance rates were initially high but then started to fall. Therefore, by the third quarter of 2010 we started to systematically check the compliance rate with the bundle.

Figure 2 shows the annual compliance rates on 4 components of the bundle before and after the implementation of this surveillance. In 2010 adherence to the elements of the care bundle was clearly suboptimal. From 725 surveyed patients, the bundle element of taking maximum barrier precautions was observed in 620 patients (86\%), changing of catheters inserted in the emergency room or in another hospital was observed in 98 patients (14\%), dedicating a line for total parenteral nutrition in 561 patients (77\%) and changing of infusion sets periodically in 724 patients (100\%). In 2011 and 2012, however, compliance with each of the elements of the bundle was 100\% (Figure 2).

Figure 3 plots the trend of monthly total compliance rates (i.e. the average of the 4 elements of the bundle of care) and the CLABSI rates throughout 2010

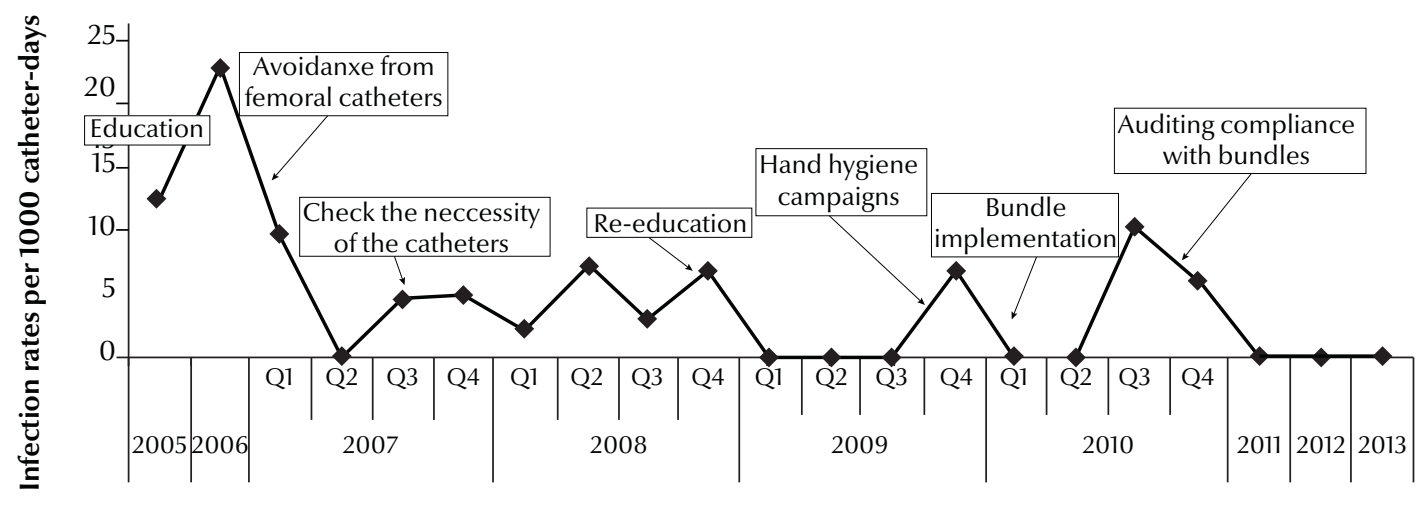

Figure 1 Central-line-associated bloodstream infection rates at Anadolu medical centre, annually and quarterly (2005-2013) 


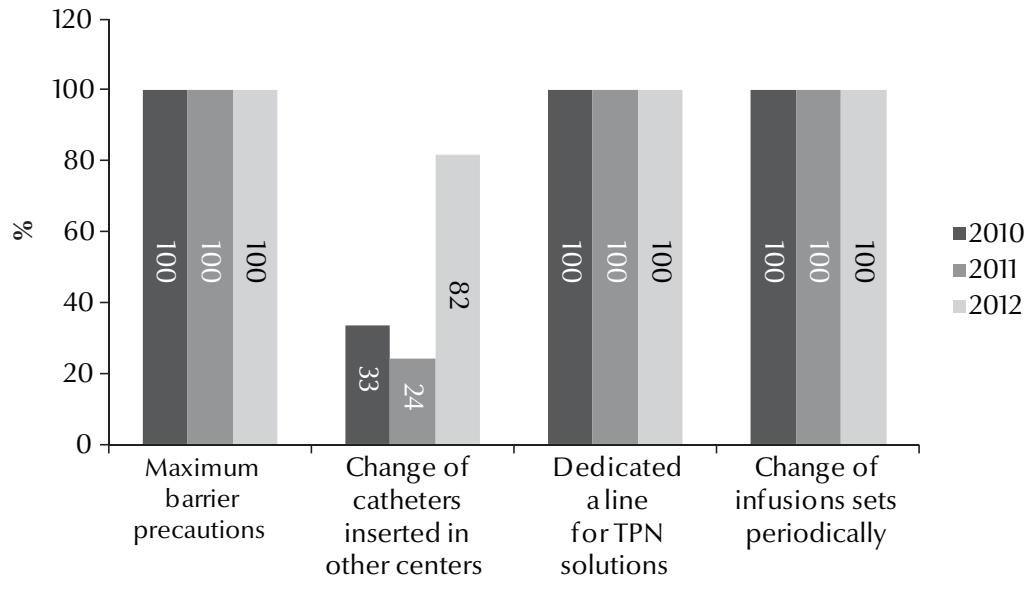

Figure 2 Compliance rates with the elements of the central-line bundle of care introduced at Anadolu medical centre (TPN = total parenteral nutrition)

and 2012 (until we stopped measuring compliance rates). The compliance rates in May to July 2010 were initially $100 \%$ and CLABSI rates were zero, but after that the compliance rate dropped. For example, compliance fell to $60 \%$ in August 2010 and remained below $100 \%$ in October and November. A corresponding rise in CLABSI rates was seen in these months from zero to 12.8 per 1000 catheter-days in August,
10.3 in September and 8.2 in October. We therefore set out to encourage and educate the staff to increase the compliance, and by the end of 2010 compliance with the bundle had returned to $100 \%$ and CLABSI rates returned to zero. Zero CLABSI rates were sustained for 3 years by auditing and maintaining compliance rates with the care bundle. Statistically, there was a strong negative correlation between bundle compliance rates and CLABSI rates $(P<0.001)$.

\section{Discussion}

This study shows that it is possible to achieve and sustain a zero rate of CLABSI in an ICU by implementing a bundle of care to prevent central-line infections; this was accompanied by encouraging a culture of patient safety in the hospital with behaviour change and adaption of health-care workers. It is important to note that not only achieving an overall CLABSI rate of zero but also sustaining this rate for a long time is a difficult and complex process. The Michigan-Keystone project in 103 ICUs in the United States of America reported a major reduction in CLABSI rates throughout the 18-month study period (5). Recently, the Matching Michigan study reported a marked reduction CLABSI rates in United Kingdom ICUs with a 2-year programme $(5,7)$. However, to our knowledge, our study will be the first published article which reports a success in creating zero catheter-related

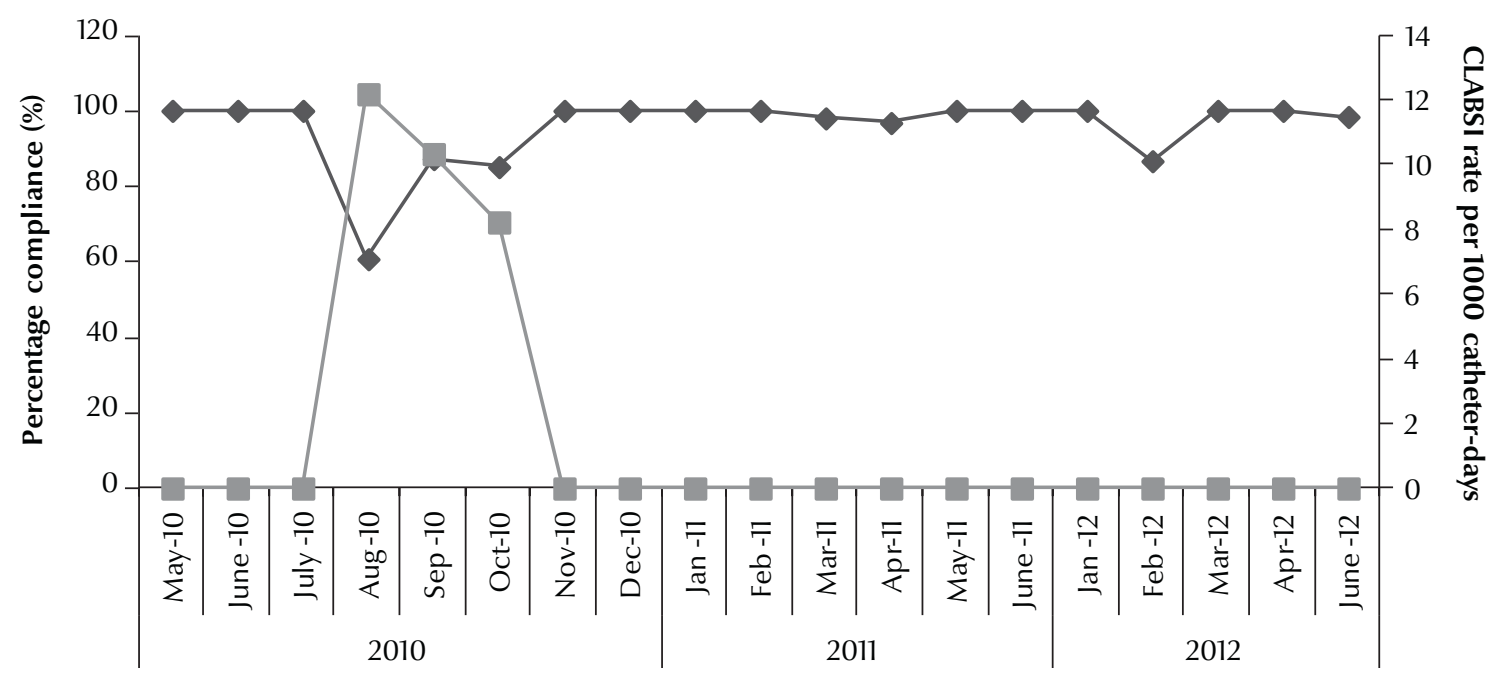

$\longrightarrow$ Bundle compliance rate

CLABSI rate per 1000 catheter-days

Figure 3 Correlation between central-line-associated bloodstream infection rates and compliance rates with the central-line bundle of care at Anadolu medical centre, monthly in the post-intervention period (Spearman correlation, $P<0.001$ ) 
infection rates for such a long period, 38 months.

Our approach to reducing the CLABSI rate in the ICU evolved over the first 6 years of our hospital's existence. Because the hospital was newly opened, all medical staff came from different centres with different habits and backgrounds. It took approximately 1 year for them to become a real team.

Insertion sites of the catheters are an important risk factor for catheter-related bloodstream infection. Skin flora at the insertion site influences the incidence of infection. Femoral catheters have a relatively high colonization rate, therefore standard guidelines recommend avoiding femoral catheter use (1). In 2006 , coinciding with an increased use of femoral catheters (21.2\%), the CLABSI rate in our hospital reached 22.9 per 1000 catheter-days. This may be explained by the young age and inexperience of internists who preferred femoral catheters. We started to encourage physicians to avoid femoral site insertion and the rate sharply decreased to 5.93 per 1000 catheter-days in 2007. Nevertheless, the infection rates in subclavian veins were lower than in jugular veins. There were two important reasons for the high usage of jugular instead of subclavian veins: the risk of arterial puncture and pneumothorax. The team was more competent in insertion of jugular lines than subclavian veins. Even though there was a decrease in CLABSI rates with certain infection control efforts before 2010, we never succeeded in totally eliminating infections. In 2010, we introduced the bundle method for prevention of CLABSI.

Strategies for prevention of CLABSI over the last decade have focused on the collective application of multifaceted bundles of care (8). The bundle method was developed by the Institute for Healthcare Improvement and subsequently the effectiveness of bundles has been validated in various trials (9-12). A bundle of care is based on evidence-based interventions or accepted consensus recommendations for care which can be easily applied using existing resources. It consists of a set structure for every event. However, as in our case, simply introducing a bundle of care does not guarantee meaningful and permanent reductions in infection rates. When the new intervention was implemented, we needed to check if there was a lack of compliance. Considering this, we started to check the compliance rates with the bundle elements and we noticed that it varied over the months. We decided to audit it systematically and intervene if there were gaps in compliance. We were able to consistently eliminate these infections from our ICU by introduction of and sustained adherence to the bundle of care.

In daily practice, omission of one or more of the bundle's components can result in failure (i.e. development of CLABSI). Our auditing process revealed such omissions in the early stages of implementation of the bundle. Through continuous assurance of high levels of adherence to the bundle we were able to achieve sustained elimination of CLABSI in our ICU.

There can be barriers to compliance, such as lack of awareness and lack of agreement with the implementation by staff. Therefore, all staff should be adequately educated and observed on their compliance with the implementation of a new strategy. A previous study has demonstrated that significant improvement in outcomes can be achieved with high compliance in implementing a care bundle (13). Furuya et al. stated that monitoring of the bundle elements and only moderate compliance is not suffcient to reduce CLABSI rates. However, in order to assure high reductions in CLABSI, compliance needs to be coupled with the ICU team's acceptance of the bundle's effectiveness (8).

In our study, with a high level of compliance we could finally achieve a zero infection rate which was sustained for 38 months. For instance, compliance with the element requiring removal or change of a catheter which had been inserted in another health centre or in the emergency room within 24 hours was only $33 \%$ at the onset of our study. With sustained focus on adherence to all elements of the bundle, this omission was eliminated. Observing and assuring compliance is important for sustained success.

According to Pronovost et al., sustainability can be achieved with a comprehensive culture of patient safety, by education, by learning from mistakes and with the engagement of senior leadership (14). Feedback can have a significant positive impact on a team (15). In our case providing feedback to the ICU team about the reduction in infection rates was an important motivator and helped to convince them of the bundle's usefulness. To foster team pride in this success, we compared our results with those of other hospitals in our meetings, rewarded the team and advertised their achievement in the hospital.

Sustainability of this success can only be possible by installing a culture of safety, rather than by temporary improvements. Patient safety culture increases the success of the intervention, but team effort and leadership engagement is crucial in installing a patient safety culture. In this regard, leadership support and role modelling are very important (16). Learning, engagement of leadership and creating a collaboration culture are important to success (17). In our study, the medical and nurse directors were very supportive. As Bosk et al. stated, changes in outcome are more complex than the simple introduction of a checklist or bundle, and reflect more complex social and cultural behaviour changes (18). Our study bears this out. The clinical bundle was introduced 6 months prior to achieving a zero rate of CLABSI. However, achievement and maintenance of success only came with the emergence of a culture of patient safety within the ICU team and their motivation on the importance of the implementation of the bundle. In a 
study of nurses' perceptions of a patient safety climate, ICU nurses are especially positive on this issue (19).

Our study focused on an ICU patient population in which central lines stayed in for a mean of 12.6 days. However, central lines have also been used in some other patient populations such those in haematology, oncology and haemodialysis settings. It may not be possible to claim a zero target in such patients. A study by McLaws et al. showed that patients with a central venous line with prolonged hospital stays were associated with a higher infection rates (20). Therefore, we agree with
Worth et al. that a zero rate of infection should be limited to ICU patients with short-term central venous catheters and this is not always applicable to high-risk groups with long stays in hospital (21).

\section{Conclusion}

With the implementation of the centralline bundle of care, sustained adherence to all of its components and a culture of patient safety installed, it was possible to achieve and maintain a zero rate of CLABSI in an ICU. In our hospital, this was achieved by engagement of medical and nursing leadership, intensive education and monitoring efforts, and ultimately motivation of the ICU team on compliance with the bundles.

\section{Acknowledgements}

This study could not be completed without active contribution of all the staff in the ICU. We would like to thank Shmuel Shoham from Johns Hopkins University for reviewing this manuscript and Firat Arkuran for assistance with statistical analyses.

\section{Funding: None.}

Competing interests: None declared.

\section{References}

1. O'Grady NP, Alexander M, Dellinger EP, Gerberding JL, Heard SO, Maki DG, et al.; Centers for Disease Control and Prevention. Guidelines for the prevention of intravascular catheter-related infections. MMWR Recomm Rep. 2002 Aug 9;51 RR-10:1-29. PMID:12233868

2. Warren DK, Quadir WW, Hollenbeak CS, Elward AM, Cox MJ, Fraser VJ. Attributable cost of catheter-associated bloodstream infections among intensive care patients in a nonteaching hospital. Crit Care Med. 2006 Aug;34(8):2084-9. PMID:16763511

3. Dudeck MA, Horan TC, Peterson KD, Allen-Bridson K, Morrell G, Pollock DA, et al. National Healthcare Safety Network (NHSN) Report, data summary for 2010, device-associated module. Am J Infect Control. 2011 Dec;39(10):798-816. PMID:22133532

4. Cardo D, Dennehy PH, Halverson P, Fishman N, Kohn M, Murphy $\mathrm{CL}$, et al.; HAI Elimination White Paper Writing Group. Moving toward elimination of healthcare-associated infections: a call to action. Am J Infect Control. 2010 Nov;38(9):671-5. PMID:21058460

5. Bion J, Richardson A, Hibbert P, Beer J, Abrusci T, McCutcheon $M$, et al.; Matching Michigan Collaboration and Writing Committee. "Matching Michigan": a 2-year stepped interventional programme to minimise central venous catheter-blood stream infections in intensive care units in England. BMJ Qual Saf. 2013 Feb;22(2):110-23. PMID:22996571

6. Pronovost P, Needham D, Berenholtz S, Sinopoli D, Chu H, Cosgrove $S$, et al. An intervention to decrease catheter-related bloodstream infections in the ICU. N Engl J Med. 2006 Dec 28;355(26):2725-32. PMID:17192537

7. Burrell AR, McLaws ML, Murgo M, Calabria E, Pantle AC, Herkes R; Central Line Associated Bacteraemia in NSW Intensive Care Units (CLAB ICU) Collaborative. Aseptic insertion of central venous lines to reduce bacteraemia. Med J Aust. 2011 Jun 6;194(11):583-7. PMID:21644871

8. Furuya EY, Dick A, Perencevich EN, Pogorzelska M, Goldmann D, Stone PW. Central line bundle implementation in US intensive care units and impact on bloodstream infections. PLoS One. 2011;6(1):e15452. PMID:21267440

9. Institute for Healthcare Improvement [Internet] (http://www.ihi. org/, accessed 9 February 2015).

10. Guerin K, Wagner J, Rains K, Bessesen M. Reduction in central line-associated bloodstream infections by implementa- tion of a postinsertion care bundle. Am J Infect Control. 2010 Aug;38(6):430-3. PMID:20570395

11. Galpern D, Guerrero A, Fahoum B, et al. Effectiveness of central line-associated infections in the intensive care unit. Surgery. 2008;144:492-5. PMID:18847630

12. Render ML, Brungs S, Kotagal U, Nicholson M, Burns P, Ellis $\mathrm{D}$, et al. Evidence-based practice to reduce central line infections. Jt Comm J Qual Patient Saf. 2006 May;32(5):253-60. PMID:16761789

13. Lisboa T, Rello J. Towards zero rate in healthcare-associated infections: one size shall not fit all.... Crit Care. 2013;17(3):139. PMID:23659634

14. Provonost PJ, Goeschel CA, Colantuoni E, Watson S, Lubomski $\mathrm{LH}$, Berenholtz SM, et al. Sustaining reduction in catheter related bloodstream infections in Michigan intensive care units: observational study. BMJ. 2010;340:c309. PMID:19084157

15. Exline MC, Ali NA, Zikri N, Mangino JE, Torrence K, Vermillion $B$, et al. Beyond the bundle-journey of a tertiary care medical intensive care unit to zero central line-associated bloodstream infections. Crit Care. 2013;17(2):R41. PMID:23497591

16. Zack J. Zeroing in on zero tolerance for central line-associated bacteremia. 2 Am J Infect Control. 2008 Dec;36(10):S176.e1-2.

17. Longmate AG, Ellis KS, Boyle L, Maher S, Cairns CJ, Lloyd SM, et al. Elimination of central-venous-catheter-related bloodstream infections from the intensive care unit. BMJ Qual Saf. 2011 Feb;20(2):174-80. PMID:21303772

18. Bosk CL, Dixon-Woods M, Goeschel CA, Pronovost PJ. Reality check for checklists. Lancet. 2009 Aug 8;374(9688):444-5 PMID:19681190

19. Ballangrud R, Hedelin B, Hall-Lord ML. Nurses' perceptions of patient safety climate in intensive care units: a cross-sectional study. Intensive Crit Care Nurs. 2012 Dec;28(6):344-54. PMID:22999498

20. McLaws ML, Burrell AR. Zero risk for central line-associated bloodstream infection: are we there yet? Crit Care Med. 2012 Feb;40(2):388-93. PMID:22020239

21. Worth LJ, McLaws ML. Is it possible to achieve a target of zero central line associated bloodstream infections? Curr Opin Infect Dis. 2012 Dec;25(6):650-7. PMID:23041775 